\title{
3D CRACK DETECTION USING AN XFEM VARIANT AND GLOBAL OPTIMIZATION ALGORTITHMS
}

\author{
KONSTANTINOS AGATHOS*, ELENI CHATZI ${ }^{\dagger}$ AND STÉPHANE P. A. BORDAS ${ }^{* \ddagger * *}$ \\ *University of Luxembourg \\ Luxembourg, Luxembourg \\ e-mail: agathosk@civil.auth.gr \\ ${ }^{\dagger}$ ETH Zürich \\ Zürich, Switzerland \\ e-mail: chatzi@ibk.baug.ethz.ch \\ ${ }^{\ddagger}$ Cardiff University \\ Cardiff, U.K. \\ e-mail: stephane.bordas@alum.northwestern.edu \\ **Adjunct Professor, The University of Western Australia \\ Australia
}

Key words: XFEM, crack detection, CMA-ES, global optimization

\begin{abstract}
In the present work, a scheme is presented for the detection of cracks in three dimensional (3D) structures. The scheme is based on the combination of a newly introduced variation of the extended finite element method (XFEM) and global optimization algorithms.
\end{abstract}

As with existing crack detection schemes, optimization algorithms are employed to minimize the norm of the difference between measured response of the structure, typically strains in some specific points along the boundary, and the response predicted numerically by XFEM. During the optimization procedure the crack geometry is parametrized and the parameters serve as design variables. The whole procedure involves the solution of a very large number of forward problems, which constitute the main computational effort. Therefore, emphasis is given in the reduction of the computational cost associated with the solution of each individual forward problem since it can directly affect the total computational time.

The employed XFEM variant can provide increased accuracy for the forward problems at a reduced computational toll, thus decreasing the overall analysis time associated with the crack detection scheme. This reduction is a result of the improved conditioning of the system matrices which leads to a decrease in the time needed to solve the corresponding systems which ranges from $40 \%$ up to a few orders of magnitude depending on the enrichment strategy used.

Since during the optimization procedure cracks are randomly generated, cracks that lie beyond the boundaries of the structure can occur. In order to exclude those cracks, implicit functions are defined in order to localize the cracks within the structure. In some cases those functions are modified so as to exclude also cracks lying in further invalid locations within the search space.

The potential of the proposed scheme is demonstrated through numerical examples involving the detection of cracks in 3D structures. 


\section{INTRODUCTION}

In recent years, extraction of feedback on structural performance by means of sensory information, also referred to as Structural Health Monitoring (SHM), has gained significant attention amongst both researchers and practitioners [1]. A significant class of SHM methods lies in the so called Nondestructive evaluation (NDE) techniques, mainly focused on detection of structural flaws, for ensuring safety and reliability of structural systems. A number of non-invasive techniques rely on the detection and interpretation of shifts in the dynamic properties of the structure, such as frequencies, mode-shapes, transfer functions and electromechanical impedance [2-9] as reported via use of ultrasonic, radiographic, thermographic, impact analysis, electrical impedance tomography and other measurement schemes. These methods come with their respective advantages and limitations depending on the application [10] and the configuration of the measurement system employed.

Independent of the configuration however, all schemes necessitate a processing algorithm tying the data acquired into effective knowledge with respect to the existence (detection), location (localization), and severity (quantification) of the flaw(s). To this end, an inverse problem is most commonly structured in which evidence of system states, such as displacements, accelerations and strains serve as the input, while the physical properties of the system and potential flaws form the unknown variables to be identified. Typically, the solution of inverse problems comprises an iterative process, where minimization of some error function is sought between the simulated and observed system states. Such a process calls for multiple solutions of a changing forward problem, which is every time reconfigured on the basis of a "candidate" set of solution variables.

A number of optimization procedures, whether deterministic or stochastic, is suited to solution of this problem, which is multimodal in nature. However, heuristic schemes lend them- selves as particularly suited to such a purpose, since they are more adept in alleviating the occurrence of local minima, while at the same time allowing for flexibility in the the adoption of the forward problem solver. Within this context, non-destructive assessment techniques have been coupled with Genetic Algorithms [11, 12], artificial Neural Networks [13], Particle Swarm Methods [14], and Machine Learning Algorithms [15]

Further to the optimization tool used however, of particular importance is the solution method employed. To this end, Rabinovich et al. [12,16] proposed a flaw detection scheme relying on application of the eXtended Finite Element Method (XFEM) and Genetic Algorithms (GAs). The authors demonstrate that cracks in flat membranes can efficiently be detected by this algorithm in both static and dynamic excitations. The use of an XFEM scheme accelerates the forward problem solution, ridding of the need for remeshing every time the geometry of the flaw is reconfigured [17-21]. In later work by Waisman et al. [22], the XFEM-GA technique was extended to account for various types of flaws such as straight cracks, circular holes, and irregular-shaped holes in elastostatic problems. The scheme was further extended in the work of Chatzi et al. [23], via fusion of an elliptical hole XFEM formulation, which allowed for further flexibility in the definition of the flaw's geometry. This work further validated efficacy of the XFEM-GA scheme via experimental studies. Finally, Sun at al. [24, 25] presented an adaptive algorithm, once again relying on XFEM, able to detect multiple flaws without prior knowledge on their number by means of an Enhanced Artificial Bee Colony (EABC) algorithm. The algorithm is based on the introduction of topological variables into the search space, used to adaptively activate/deactivate flaws during run time until convergence is reached.

A drawback of all aforementioned works lies in their limitation to the two dimensional domain. In recent work of the authoring team, 
Agathos et al. [26, 27] introduced a variation of the extended finite element method for threedimensional fracture mechanics. A novel form of enrichment is developed and special techniques are employed for matching displacements of the standard and enriched elements in order to achieve higher accuracy, optimal convergence rates, and improved conditioning for two-dimensional and three-dimensional crack problems. The efficacy of the method in implementation in the 3D domain, which is in essence the true domain of most practical situations, renders this scheme ideal for adoption within the inverse problem formulation discussed earlier. The 3D XFEM solution tool is herein coupled with a Covariance Matrix Adaptation Evolution Strategy (CMA-ES) [28] optimization tool, maintaining the benefits of stochastic, derivative-free optimization methods inspired by biological processes.

The outline of this paper is as follows. In the following section the inverse problem is formulated, in section 3 the forward problem as well as the solution tool are described. In section 4 some details are given regarding the parametrization of the problem and the way constraints are handled while in section 5 the efficiency of the proposed crack detection scheme is demonstrated in a 3D example. Finaly, in section 6 some conclusions are drawn.

\section{INVERSE PROBLEM}

In general, the problem studied in the present work consists of detecting flaws in an existing structure given some measured response of the structure and some specific loads. In particular, the flaws to be detected are cracks and the measured response for the purposes of this work is strains at some given points.

Mathematically, the above is formulated as an optimization problem as in Reference [22]:

$$
\begin{aligned}
& \text { Find } \beta_{i} \text { such that } \\
& \mathcal{F}\left(r\left(\beta_{i}\right)\right) \rightarrow \min
\end{aligned}
$$

where $\beta_{i}$ are parameters describing the crack geometry, $r$ is a norm of the difference (resid- ual) between the measured and the computed response of the structure and $\mathcal{F}$ is some function of this residual.

As mentioned in section 1, the above problem is solved using a Covariance Matrix Adaptation Evolution Strategy (CMA-ES) [28-30].

\section{SOLUTION OF THE FORWARD PROBLEM WITH XFEM}

During the optimization process required for the solution of the inverse problem, the forward problem has to be solved several times for different values of the design variables. As a result an accurate and reliable method for the solution of the forward problem is necessary. In the following subsections, the forward problem is mathematically formulated and the solution method is presented.

\subsection{Problem statement}

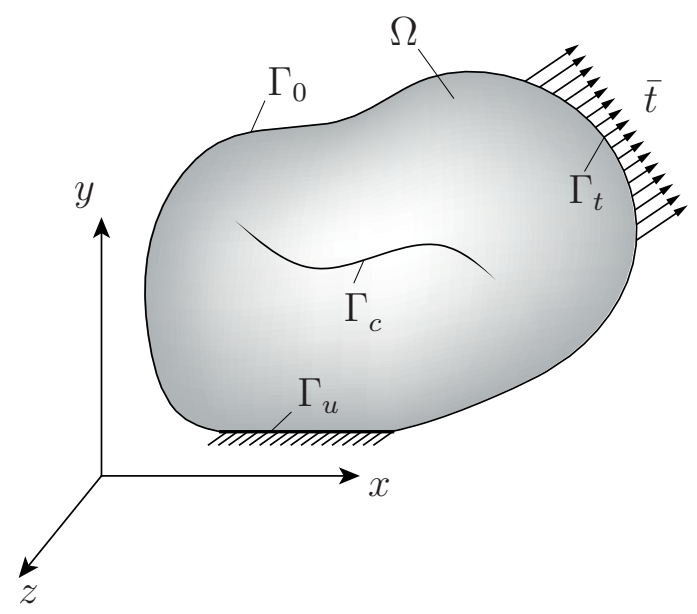

Figure 1: Cracked Body and boundary conditions.

The problem consists of a cracked linear elastic solid $\Omega$ (Figure 11) bounded by the boundary $\Gamma$ where:

$$
\Gamma=\Gamma_{0} \cup \Gamma_{u} \cup \Gamma_{t} \cup \Gamma_{c}
$$

$\Gamma_{0}$ is the part of the boundary where no boundary conditions are applied.

$\Gamma_{u}$ is the part of the boundary where displacements $\bar{u}$ are imposed as Dirichlet boundary conditions. 
$\Gamma_{t}$ is the part of the boundary where surface tractions $\bar{t}$ are applied as Neumann conditions.

$\Gamma_{c}$ is the crack surface.

The weak form of the equilibrium equations is formulated as:

Find a kinematically admissible displacement field $\boldsymbol{u} \in \mathcal{U}$ such that $\forall \boldsymbol{v} \in \mathcal{V}$

$$
\int_{\Omega} \boldsymbol{\epsilon}(\boldsymbol{u}): \boldsymbol{D}: \boldsymbol{\epsilon}(\boldsymbol{v}) d \Omega=\int_{\Omega} \boldsymbol{b} \cdot \boldsymbol{v} d \Omega+\int_{\Gamma_{t}} \overline{\boldsymbol{t}} \cdot \boldsymbol{v} d \Gamma
$$

where :

$$
\mathcal{U}=\left\{\boldsymbol{u} \mid \boldsymbol{u} \in\left(H^{1}(\Omega)\right)^{3}, \boldsymbol{u}=\overline{\boldsymbol{u}} \text { on } \Gamma_{u}\right\}
$$

and

$$
\mathcal{V}=\left\{\boldsymbol{v} \mid \boldsymbol{v} \in\left(H^{1}(\Omega)\right)^{3}, \boldsymbol{v}=\mathbf{0} \text { on } \Gamma_{u}\right\}
$$

Functions of $H^{1}(\Omega)$ are implicitly discontinuous along the crack surface.

In the above, $\boldsymbol{\epsilon}$ is the small strain field, $\boldsymbol{D}$ is the elasticity tensor and $\boldsymbol{b}$ is the applied body force per unit volume.

\subsection{Crack representation}

In the present work cracks are represented implicitly using the level set method, which is a common practice in XFEM [31-33]. Level set functions are denoted as $\phi$ and $\psi$ and for an arbitrary point $\mathrm{x}$ they are defined as follows:

- $\phi(\mathbf{x})$ is the signed distance from the crack surface defined as:

$$
\phi(\mathbf{x})=\min _{\overline{\mathbf{x}} \in \Gamma_{c}}\|\mathbf{x}-\overline{\mathbf{x}}\| \operatorname{sign}\left(\mathbf{n}^{+} \cdot(\mathbf{x}-\overline{\mathbf{x}})\right)
$$

where $\mathbf{n}^{+}$is the outward normal to the crack surface and sign () is the sign function.

- $\psi(\mathbf{x})$ is a signed distance function such that $\nabla \phi \cdot \nabla \psi=0$ and $\phi(\mathbf{x})=0$ and $\psi(\mathbf{x})=0$ defines the crack front.
Additionally, a polar coordinate system is defined along the crack front with coordinates [31-33]:

$$
r=\sqrt{\phi^{2}+\psi^{2}}, \quad \theta=\arctan \left(\frac{\phi}{\psi}\right)
$$

These coordinates refer to a plane normal to the crack front.

\subsection{Discretization}

The weak form presented above is discretized using XFEM [17] and more specifically, the variation of the method introduced in the authors' previous works [26, 27].

In XFEM the FE approximation is enriched using functions that are able to represent known features of the solution. Enrichment is realized by employing the partition of unity (PU) method [34]:

$$
\mathbf{u}(\mathbf{x})=\underbrace{\sum_{\forall I} N_{I}(\mathbf{x}) \mathbf{u}_{I}}_{\text {FE approximation }}+\underbrace{\sum_{\forall I} N_{I}^{*}(\mathbf{x}) \Psi(\mathbf{x}) \mathbf{b}_{I}}_{\text {enriched part }}
$$

where $N_{I}(\mathbf{x})$ are the FE interpolation functions, $\mathbf{u}_{I}$ are $\mathrm{FE}$ degrees of freedom (dofs), $N_{I}^{*}(\mathbf{x})$ is a basis of functions that form a partition of unity, $\Psi(\mathbf{x})$ are the enrichment functions and $\mathbf{b}_{I}$ are the enriched degrees of freedom.

The enrichment functions most commonly used for cracks are the modified Heavyside or jump enrichment functions:

$$
H(\phi)=\left\{\begin{aligned}
1 & \text { for } \phi>0 \\
-\quad 1 & \text { for } \phi<0
\end{aligned}\right.
$$

which are used to represent the displacement jump along the crack surfaces, and the asymptotic or tip enrichment functions:

$$
\begin{aligned}
F_{j}(r, \theta)= & {\left[\sqrt{r} \sin \frac{\theta}{2}, \sqrt{r} \cos \frac{\theta}{2}, \ldots\right.} \\
& \left.\ldots \sqrt{r} \sin \frac{\theta}{2} \sin \theta, \sqrt{r} \cos \frac{\theta}{2} \sin \theta\right]
\end{aligned}
$$


which are used to represent the asymptotic fields around the crack front.

Since enrichment is applied locally, the elements where each enrichment function is used have to be appropriately selected:

- Jump enrichment is used for elements that are divided in two parts by the crack surface.

- Tip enrichment is used for elements that contain the crack front (topological enrichment), of for elements that lie in a certain distance (enrichment radius) from the crack front (geometrical enrichment). In the first case a lack of optimal convergence is observed [35, 36], while in the second, although convergence is optimal, conditioning problems are observed for the solution of which, special techniques have been developed [36-38].

The functions $N_{I}^{*}(\mathbf{x})$ used for the partition of unity enrichment are typically selected to coincide with the FE shape functions $\left(N_{I}(\mathbf{x}) \equiv\right.$ $N_{I}^{*}(\mathbf{x})$ ). In the variant used in the present work however, an alternative definition is given which has been shown ( [26, 27]) to provide improved conditioning of the resulting stiffness matrices, and as a result enable the use of geometrical enrichment in 3D XFEM. More specifically, a superimposed mesh of special elements is used to discretize the crack front as illustrated in Figure 2 and the shape functions corresponding to those front elements are used as a basis for the PU enrichment.

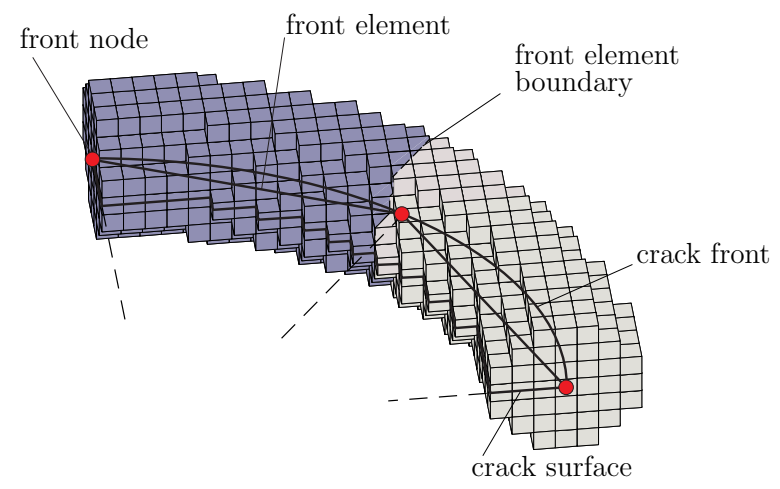

Figure 2: Front elements, nodes and boundaries.
The shape functions of the front elements are defined as:

$$
\mathbf{N}^{g}(\xi)=\left[\begin{array}{ll}
\frac{1-\xi}{2} & \frac{1+\xi}{2}
\end{array}\right]
$$

where $\xi$ is the local coordinate of the superimposed element (Figure 3). More details for the definition of this parameter are given in References [26, 27]

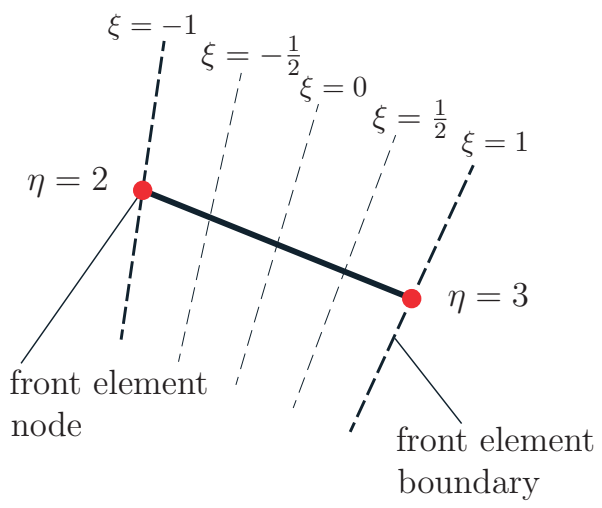

Figure 3: Local coordinate $\xi$ of the front elements.

In order to deal with blending problems between the standard and the enriched part of the approximation [39.-44], the techniques developed in the works of Fries [40] and Ventura et al. [43] are employed as in our previous works [26, 27]. Those techniques involve the definition of a weight function $\varphi(\mathbf{x})$ that assumes a value of 1 for the fully enriched elements and linearly fades to zero for the blending elements. The area along which the weight function fades to zero can be composed by one or several layers of elements [27].

The displacement approximation for the method is: 


$$
\begin{aligned}
& \mathbf{u}(\mathbf{x})=\sum_{I \in \mathcal{N}} N_{I}(\mathbf{x}) \mathbf{u}_{I}+ \\
& +\bar{\varphi}(\mathbf{x}) \sum_{J \in \mathcal{N}^{j}} N_{J}(\mathbf{x})\left(H(\mathbf{x})-H_{J}\right) \mathbf{b}_{J}+ \\
& +\varphi(\mathbf{x})\left(\sum_{K \in \mathcal{N}^{s}} N_{K}^{g}(\mathbf{x}) \sum_{j} F_{j}(\mathbf{x})-\right. \\
& \left.-\sum_{T \in \mathcal{N}^{t}} N_{T}(\mathbf{x}) \sum_{K \in \mathcal{N}^{s}} N_{K}^{g}\left(\mathbf{x}_{T}\right) \sum_{j} F_{j}\left(\mathbf{x}_{T}\right)\right) \mathbf{c}_{K j}
\end{aligned}
$$

where:

$\mathcal{N}$ is the set of all nodes in the FE mesh.

$\mathcal{N}^{j}$ is the set of jump enriched nodes. This nodal set includes all nodes whose support is split in two by the crack and in addition belong to elements where the weight function $\bar{\varphi}(\mathbf{x})$ assumes values greater than zero.

$\mathcal{N}^{t}$ is the set of tip enriched nodes. This nodal set includes all nodes that belong to an element with at least one node inside the enrichment radius.

$\mathcal{N}^{s}$ is the set of nodes in the superimposed mesh.

In Reference [27] the proposed method has been showed to provide increased accuracy at a reduced computational cost which in the present application is of great importance given the fact that the solution of the forward problems represents the biggest part of the total computational time.

\section{PROBLEM PARAMETRIZATION AND CONSTRAINTS}

\subsection{Parametrization}

As far as parametrization of the crack geometry is concerned, a simple approach is employed in which crack shapes are approximated by ellipses. This choice is justified by the fact that by employing ellipses, a variety of geometrical shapes can be roughly approximated using a relatively small number of parameters.

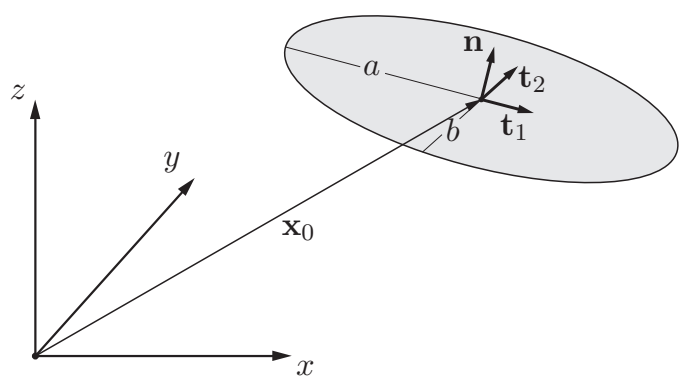

Figure 4: Parametrization of an elliptical crack.

As shown in Figure 4 the parameters involved in the definition of an elliptical crack are the coordinates of its center point $\mathbf{x}_{0}$ $\left(\left\{x_{0}, y_{0}, z_{0}\right\}\right)$, the angles of rotation about the three axes $\theta_{x}, \theta_{y}$ and $\theta_{z}$ which are needed to produce vectors $\mathbf{n}, \mathbf{t}_{1}$ and $\mathbf{t}_{2}$, and lengths $a$ and $b$.

During the optimization procedure, the values of those parameters that minimize the difference of the computed with the measured response of the structure should be obtained. However, the geometrical parameters of the ellipse are not used directly as design parameters. Instead, the following parameter encoding is employed:

$$
p_{i}=\frac{p_{i_{1}}+p_{i_{2}}}{2}+\frac{p_{i_{2}}-p_{i_{1}}}{2} \sin \left(\frac{\beta_{i}}{10} \cdot \frac{\pi}{2}\right)
$$

where $p_{i}$ are geometrical parameters of the crack, $p_{i_{1}}$ and $p_{i_{2}}$ are lower and upper bounds to the values of the parameters, $\beta_{i}$ are the design parameters involved in the optimization procedure (Equation 1).

Values of the design parameters are randomly generated by the optimization algorithm and their values could exceed some predefined acceptable limits, for instance cracks of very small or very large size could be generated which would cause problems in the solution of the forward problems. Consequently, the encoding of Equation 13 is required to bound the design variables within the desirable limits. 


\subsection{Constraints}

Since a very large part (more than $95 \%$ ) of the computational time is spent for the solution of forward problems, and since the values some of the parameters for the forward problems are randomly generated, it is very important to ensure that only forward problems with valid input parameters are solved. An example of invalid input parameters would be crack geometries that lie outside the structure considered. Of course, such cracks would produce large fitness function values and as a result would not be selected by the algorithm in order to create the next generation. Nevertheless, it is preferable to have a means of determining a priori whether a crack is valid and assign a large value to the fitness function since using this approach, the time consuming solution of the forward problem is avoided. An additional possibility, which is exploited in the present work, consists of locating and re-sampling invalid cracks.

For structures of very simple geometries the above procedure can be avoided by bounding the design variables as described in the previous subsection and therefore ensuring that the crack will remain within the structure geometry. For more complex geometries however, simple bounding of the variables is insufficient and a means of determining the the location of generated cracks relative to the structure is necessary. In the present work, implicit functions, and more specifically radial basis functions [45], are introduced which describe the structure boundaries. Those functions are defined so as to assume negative values for points inside the structure, positive values for points outside the structure and a value of zero for points lying on the structure boundaries.

Moreover, since the cracks considered are of elliptical shape, several points on the crack surface have to be tested in order to determine if the crack lies completely or partially outside the structure. Cracks that intersect the boundaries of the structure are not considered invalid provided that the part of the crack that lies inside the structure is large enough.

\section{NUMERICAL EXAMPLES}

\subsection{Penny crack in a cube}

The example considered consists of a penny crack in a unit cube. As illustrated in Figure 5 the cube is fixed in one side and subjected to uniform normal loads on the other side. The geometrical parameters of the problem are $L_{x}=L_{y}=L_{z}=1$ unit, $a=b=0.25$ units, while the crack lies in the center of the cube $\left(L_{x} / 2, L_{y} / 2, L_{z} / 2\right)$ and is parallel to the fixed face of the cube. For the forward problems a structured mesh of $25 \times 25 \times 25$ tetrahedral elements is used. For the discretization of the crack front involved in the proposed method the length of the front elements was set to $3 h$, where $h$ is the mesh parameter.

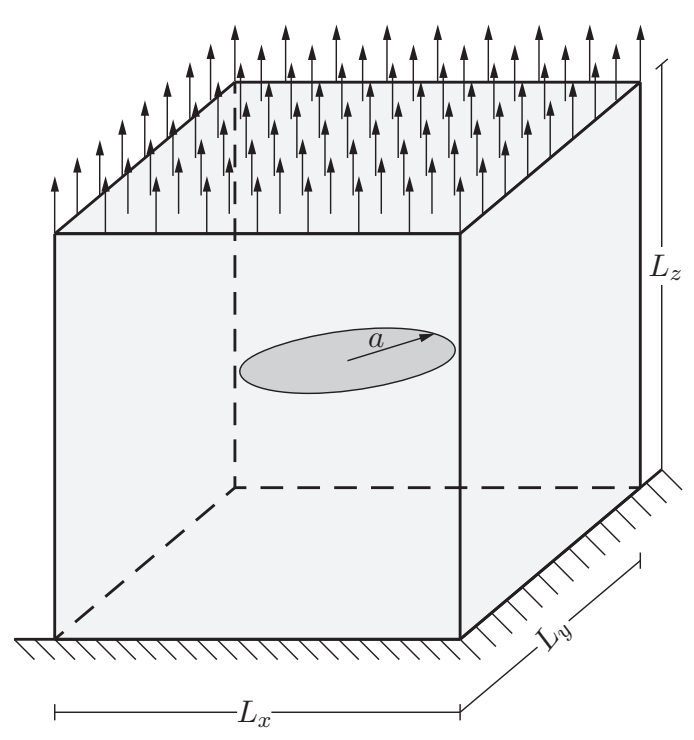

Figure 5: Penny crack in a cube geometry.

The measured response of the structure is obtained by placing sensors in the free faces of the cube in the locations displayed in Figure 6. Measurements are simulated using a finer mesh consisting of $51 \times 51 \times 51$ tetrahedral elements. 


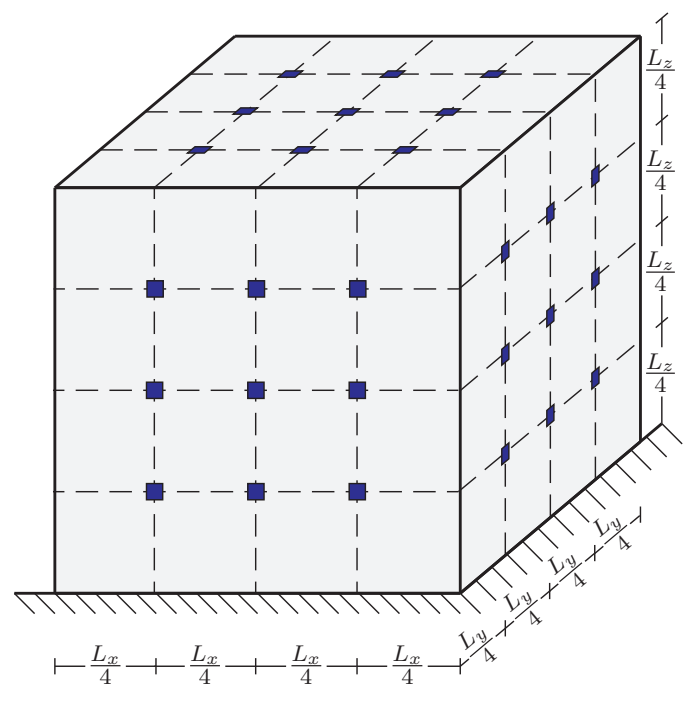

- Sensor locations

Figure 6: Penny crack in a cube, sensor locations.

The upper and lower bounds for the geometrical parameters of the crack during the optimization process were defined as $x_{0_{1}}=0$ units, $x_{0_{2}}=1$ units, $y_{0_{1}}=0$ units, $y_{0_{2}}=1$ units, $z_{0_{1}}=0$ units, $z_{0_{2}}=1$ units, $\theta_{x_{1}}=0, \theta_{x_{2}}=\pi$, $\theta_{y_{1}}=0, \theta_{y_{2}}=\pi, \theta_{z_{1}}=0, \theta_{z_{2}}=\pi, a_{1}=0.20$ units, $a_{2}=0.50$ units, $b_{1}=0.20$ units and $b_{2}=0.35$ units.

The CMA-ES algorithm was set to run for 2000 forward problem solutions and the population size was set to 20. Parameter $\sigma$ was given a value of 3 . The algorithm was run several times and some representative results are shown next.

In Figure 7 fitness function values are plotted against the number of solutions of the forward problem, while in Figures 8, 9 and 10 the best solution of the current generation is illustrated at different stages of the optimization procedure. As can be seen in Figure 10 the final solution is almost indistinguishable from the actual crack. Nevertheless, crack locations can only be accurately determined when enough measurements are available otherwise the problem becomes ill-conditioned and the optimization algorithm is not able to converge. It should also be remarked that the value of the fitness function for the final solution obtained by the optimization algorithm is slightly smaller than the value of the fitness function evaluated for the actual crack, which was also observed in Reference [16].

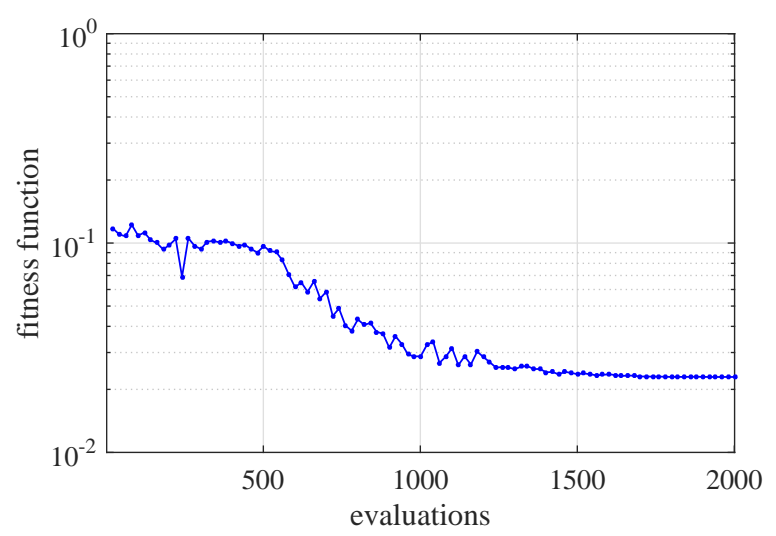

Figure 7: Convergence of the optimization problem.

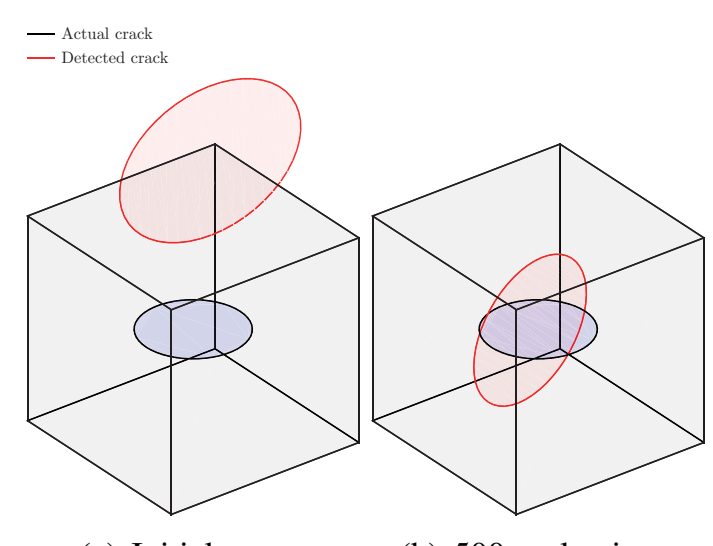

(a) Initial guess.

(b) 500 evaluations.

Figure 8: Best solution in different stages of the optimization process.

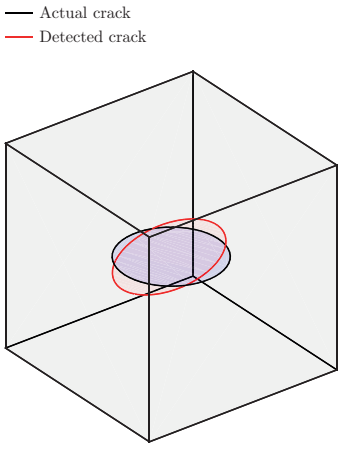

(a) 1000 evaluations.

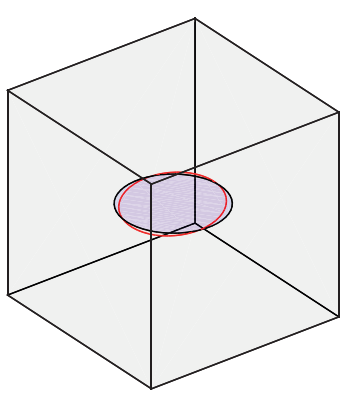

(b) 1500 evaluations.
Figure 9: Convergence of the optimization problem. 


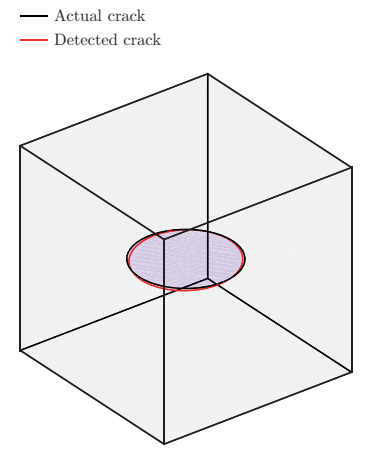

(a) Final solution.

Figure 10: Convergence of the optimization problem.

\section{CONCLUSIONS}

A three dimensional crack detection scheme based on a variation of XFEM [26, 27] and on the CMA-ES [28] algorithm was presented. The proposed scheme is a first attempt to extend existing XFEM based flaw detection schemes [16, 22, 25] in elastostatics to three dimensions. The results obtained in Section 5 are promising, however the application to larger, more realistic problems, would increase the size of the forward problems and therefore the total computational cost to the point where the scheme would be of no practical use. As a result, future work could be oriented toward the reduction of the computational cost associated with the solution of the forward problems.

\section{REFERENCES}

[1] CR Farrar and K Worden. Structural Health Monitoring: A Machine Learning Perspective. John Wiley \& Sons, Ltd, 2012.

[2] PC Chang and S C Liu. Recent research in nondestructive evaluation of civil infrastructures. Journal of Materials In Civil Engineering, 15(3):298-304, MayJune 2003.

[3] V Giurgiutiu, A Reynolds, and CA Rogers. Experimental investigation of $\mathrm{e} / \mathrm{m}$ impedance health monitoring for spot-welded structural joints. Journal Of Intelligent Material Systems And
Structures, 10(10):802-812, October 1999.

[4] WM Ostachowicz. Damage detection of structures using spectral finite element method. Comput. Struct., 86(3-5):454462, 2008.

[5] V Giurgiutiu and A Zagrai. Damage detection in thin plates and aerospace structures with the electro-mechanical impedance method. Structural Health Monitoring, 4(2):99-118, 2005.

[6] S Park, CB Yun, Y Roh, and JJ Lee. Health monitoring of steel structures using impedance of thickness modes at pzt patches. Smart Structures And Systems, 1(4):339-353, October 2005.

[7] L Borcea. Electrical impedance tomography. Inverse Problems, 18:r99-r136, 2002.

[8] Z Chaudhry, F Lalande, A Ganino, and C Rogers. Monitoring the integrity of composite patch structural repair via piezoelectric actuators/sensors. Proceedings of the AIAA/ASME/AHS/ASC 36th SDM Conference, 4:2243-48, 1995.

[9] G Park, H Sohn, C R Farrar, and D Inman. Overview of piezoelectric impedance-based health monitoring and path forward. The Shock and Vibration Digest, 35(6):451-463, 2003.

[10] CJ Hellier. Handbook of Nondestructive Evaluation. McGraw-Hill: New York, 2001.

[11] O Hunaidi. Evolution-based genetic algorithms for analysis of non-destructive surface wave tests on pavements. $\{N D T\} \&$ E International, 31(4):273 - 280, 1998. Non-destructive Testing in Civil Engineering.

[12] D Rabinovich, D Givoli, and S Vigdergauz. Crack identification by arrival time 
using xfem and a genetic algorithm. International Journal for Numerical Methods in Engineering, 2008.

[13] SJ Farley, JF Durodola, NA Fellows, and LH Hernndez-Gmez. High resolution non-destructive evaluation of defects using artificial neural networks and wavelets. $\{N D T\} \& E$ International, 52:69 - 75, 2012.

[14] W Lee, C Sun, and C Chiu. Nondestructive evaluation of buried dielectric cylinders by asynchronous particle swarm optimization. Journal of Testing and Evaluation, 43(1):212-220, 2014.

[15] A Bernieri, L Ferrigno, M Laracca, and M Molinara. Crack shape reconstruction in eddy current testing using machine learning systems for regression. IEEE Transactions on Instrumentation and Measurement, 57(9):1958-1968, Sept 2008.

[16] D Rabinovich, D Givoli, and S Vigdergauz. Xfem-based crack detection scheme using a genetic algorithm. International Journal for Numerical Methods in Engineering, 71(9):1051-1080, 2007.

[17] N Moës, J Dolbow, and T Belytschko. A finite element method for crack growth without remeshing. International Journal for Numerical Methods in Engineering, 46(1):131-150, 1999.

[18] T Belytschko and T Black. Elastic crack growth in finite elements with minimal remeshing. International Journal for $\mathrm{Nu}$ merical Methods in Engineering, 620(July 1998):601-620, 1999.

[19] N Sukumar, DL Chopp, N Moës, and $\mathrm{T}$ Belytschko. Modeling holes and inclusions by level sets in the extended finite element method. Computer Methods in Applied Mechanics and Engineering, 190(46-47):6183-6200, 2000.
[20] C Daux, N Moës, J Dolbow, N Sukumar, and T Belytschko. Arbitrary branched and intersecting cracks with the extended finite element method. International Journal for Numerical Methods in Engineering, 48(12):1741-1760, 2000.

[21] N Sukumar, N Moës, B Moran, and $\mathrm{T}$ Belytschko. Extended finite element method for three-dimensional crack modelling. International Journal for Numerical Methods in Engineering, 48(November 1999):1549-1570, 2000.

[22] H Waisman, E Chatzi, and AW Smyth. Detection and quantification of flaws in structures by the extended finite element method and genetic algorithms. International Journal for Numerical Methods in Engineering, 82(3):303-328, 2010.

[23] EN Chatzi, B Hiriyur, H Waisman, and A W Smyth. Experimental application and enhancement of the xfem-ga algorithm for the detection of flaws in structures. Computers \& Structures, 89(7):556-570, 2011.

[24] H Sun, H Waisman, and R Betti. Nondestructive identification of multiple flaws using xfem and a topologically adapting artificial bee colony algorithm. International Journal for Numerical Methods in Engineering, 95(10):871-900, 2013.

[25] Hao Sun, Haim Waisman, and Raimondo Betti. A multiscale flaw detection algorithm based on xfem. International Journal for Numerical Methods in Engineering, 100(7):477-503, 2014.

[26] K Agathos, E Chatzi, SPA Bordas, and D Talaslidis. A well-conditioned and optimally convergent xfem for $3 \mathrm{~d}$ linear elastic fracture. International Journal for $\mathrm{Nu}$ merical Methods in Engineering, 2015.

[27] K Agathos, E Chatzi, and S Bordas. Stable $3 \mathrm{~d}$ extended finite elements with higher 
order enrichment for accurate non planar fracture. Computer Methods in Applied Mechanics and Engineering, 2016 (under review).

[28] N Hansen, SD Muller, and P Koumoutsakos. Reducing the time complexity of the derandomized evolution strategy with covariance matrix adaptation (CMAES). Evolutionary Computation, 11(1):118, 2003.

[29] N Hansen and S Kern. Evaluating the cma evolution strategy on multimodal test functions. In Parallel problem solving from nature-PPSN VIII, pages 282-291. Springer, 2004.

[30] R Ros and N Hansen. A simple modification in cma-es achieving linear time and space complexity. In Parallel Problem Solving from Nature-PPSN X, pages 296-305. Springer, 2008.

[31] N Moës, A Gravouil, and T Belytschko. Non-planar 3D crack growth by the extended finite element and level sets-Part I: Mechanical model. International Journal for Numerical Methods in Engineering, 53(11):2549-2568, April 2002.

[32] A Gravouil, N Moës, and T Belytschko. Non-planar 3D crack growth by the extended finite element and level sets-Part II: Level set update. International Journal for Numerical Methods in Engineering, 53(11):2569-2586, April 2002.

[33] M Duflot. A study of the representation of cracks with level sets. International Journal for Numerical Methods in Engineering, 70(November 2006):1261-1302, 2007.

[34] JM Melenk and I Babuska. The partition of unity finite element method: basic theory and applications. Computer methods in applied mechanics and engineering, 139(1-4):289-314, 1996.
[35] FL Stazi, E Budyn, J Chessa, and T Belytschko. An extended finite element method with higher-order elements for curved cracks. Computational Mechanics, 31(1-2):38-48, 2003.

[36] P Laborde, J Pommier, Y Renard, and M Salaün. High-order extended finite element method for cracked domains. International Journal for Numerical Methods in Engineering, 64(3):354-381, September 2005 .

[37] E Béchet, H Minnebo, N Moës, and B Burgardt. Improved implementation and robustness study of the X-FEM for stress analysis around cracks. International Journal for Numerical Methods in Engineering, 64(8):1033-1056, October 2005.

[38] A Menk and SPA Bordas. A robust preconditioning technique for the extended finite element method. International Journal for Numerical Methods in Engineering, 85(October 2010):1609-1632, 2011.

[39] J Chessa, H Wang, and T Belytschko. On the construction of blending elements for local partition of unity enriched finite elements. International Journal for Numerical Methods in Engineering, 57(7):10151038, June 2003.

[40] TP Fries. A corrected XFEM approximation without problems in blending elements. International Journal for Numerical Methods in Engineering, 75(November 2007):503-532, 2008.

[41] R Gracie, H Wang, and T Belytschko. Blending in the extended finite element method by discontinuous Galerkin and assumed strain methods. International Journal for Numerical Methods in Engineering, 74(November 2007):1645-1669, 2008. 
[42] JE Tarancón, A Vercher, E Giner, and FJ Fuenmayor. Enhanced blending elements for XFEM applied to linear elastic fracture mechanics. International Journal for Numerical Methods in Engineering, 77(July 2008):126-148, 2009.

[43] G Ventura, R Gracie, and T Belytschko. Fast integration and weight function blending in the extended finite element method. International journal for numerical methods in engineering, 77(July 2008):1-29, 2009.

[44] S Loehnert, DS Mueller-Hoeppe, and
P Wriggers. 3D corrected XFEM approach and extension to finite deformation theory. International Journal for Numerical Methods in Engineering, 86(October 2010):431-452, 2011.

[45] JC Carr, RK Beatson, JB Cherrie, TJ Mitchell, WR Fright, BC McCallum, and TR Evans. Reconstruction and representation of $3 \mathrm{~d}$ objects with radial basis functions. In Proceedings of the 28th annual conference on Computer graphics and interactive techniques, pages 67-76. ACM, 2001. 